The Astrophysical Journal, 562:L173-L175, 2001 December 1

(C) 2001. The American Astronomical Society. All rights reserved. Printed in U.S.A.

\title{
A YOUNG PROTOPLANETARY DISK IN THE BOK GLOBULE CB 26?
}

\author{
R. LAunhardt and A. I. SARgent \\ Division of Physics, Mathematics, and Astronomy, MS 103-33, California Institute of Technology, Pasadena, CA 91125; rl@astro.caltech.edu \\ Received 2001 September 3; accepted 2001 October 19; published 2001 November 7
}

\begin{abstract}
We present subarcsecond resolution millimeter-wave images of a circumstellar disk in the Bok globule CB 26. The presence of an edge-on disk is confirmed by the dust continuum morphology and the velocity field of ${ }^{13} \mathrm{CO}$ emission, which displays a Keplerian rotation pattern about an axis perpendicular to the long axis of the dust emission. We deduce a mass of $\sim 0.3 M_{\odot}$ for the obscured central star. The disk is optically thick at millimeter wavelengths inside $120 \mathrm{AU}$, has a symmetric $20^{\circ}$ warp beyond $120 \mathrm{AU}$, an outer radius of $200 \mathrm{AU}$, and a mass of at least $0.1 M_{\odot}$. We suggest that the CB 26 system is in an intermediate stage between deeply embedded protostellar accretion disks and the more evolved, perhaps protoplanetary, disks around T Tauri stars.
\end{abstract}

Subject headings: circumstellar matter — dust, extinction - ISM: globules —

planetary systems: protoplanetary disks — stars: formation

\section{INTRODUCTION}

CB 26 (L1439) is a small cometary-shaped Bok globule located $\sim 10^{\circ}$ north of the Taurus/Auriga dark cloud. Launhardt \& Henning (1997) suggested a distance of 300 pc. From a reexamination of the larger scale velocity structure (Ungerechts \& Thaddeus 1987), we believe it is part of the Taurus/Auriga complex at $140 \mathrm{pc}$. Single-dish submillimeter images at $10^{\prime \prime}$ resolution show strong unresolved dust continuum emission at the southwest rim of the globule, surrounded by a thin asymmetric envelope with a well-ordered magnetic field directed along P.A. $\sim 25^{\circ}$ (Henning et al. 2001). A small bipolar nearinfrared (NIR) nebula bisected by a dark extinction lane is associated with the millimeter emission. The subarcsecond resolution NIR polarization pattern is consistent with the presence of an almost edge-on circumstellar disk elongated along the extinction lane at P.A. $\sim 60^{\circ}$ and a thin envelope (Stecklum et al. 2001). The spectral energy distribution indicates a low-mass, $\geq 0.5 L_{\odot}$ pre-main-sequence (PMS) star surrounded by a disk and an envelope (Stecklum et al. 2001), i.e., a Class I object (Adams, Lada, \& Shu 1987; Kenyon \& Hartmann 1995).

\section{OBSERVATIONS AND DATA REDUCTION}

CB 26 was observed with the Owens Valley Radio Observatory millimeter-wave array between 2000 January and December. Three configurations of the six $10.4 \mathrm{~m}$ antennas provided baselines in the range of $6-180 \mathrm{k} \lambda$ at $2.7 \mathrm{~mm}(110 \mathrm{GHz})$ and $12-400 \mathrm{k} \lambda$ at $1.3 \mathrm{~mm}(232 \mathrm{GHz})$. Average single-sideband system temperatures of the SIS receivers were 300-400 K at $110 \mathrm{GHz}$ and $300-600 \mathrm{~K}$ at $236 \mathrm{GHz}$. The digital correlator was centered on the ${ }^{13} \mathrm{CO}(1-0)$ line at $110.2 \mathrm{GHz}$, adopting the systemic velocity of CB $26, v_{\mathrm{LSR}}=5.5 \mathrm{~km} \mathrm{~s}^{-1}$; the spectral resolution and bandwidth were 0.17 and $5 \mathrm{~km} \mathrm{~s}^{-1}$, respectively. Continuum emission at 1.3 and $2.7 \mathrm{~mm}$ was observed simultaneously in $2 \mathrm{GHz}$ wide bands, except in the highest resolution configuration, where the $4 \mathrm{GHz}$ wide-band capability of new $1 \mathrm{~mm}$ receivers was used. Amplitude and phase calibration relied on frequent observations of a nearby quasar, resulting in an absolute position uncertainty of 0 "'2. Flux densities are based on observations of Uranus and Neptune, with resulting uncertainties of $20 \%$. The raw data were calibrated and edited using the MMA software package (Scoville et al. 1993). Mapping and data analysis used the MIRIAD toolbox (Sault, Theuben,
\& Wright 1995). We also applied maximum entropy deconvolution (the maximum entropy method [MEM]) to the data and obtained images that are very similar to those obtained by standard cleaning.

\section{RESULTS}

In Figure 1, our dust continuum images of CB 26 show a source elongated along P.A. $=60^{\circ} \pm 5^{\circ}$. At $2.7 \mathrm{~mm}$, the projected length derived from the distribution of clean components is $230 \pm 30 \mathrm{AU}$. The minor axis is unresolved, and there is no hint of an envelope. By contrast, the $1.3 \mathrm{~mm}$ images show a narrow central structure of length $400 \pm 40 \mathrm{AU}$ and a small envelope of size $220 \times 280$ AU. The scale height $h$ of the central structure remains unresolved, i.e., $h \leq 20$ AU. Beyond radii of $120 \mathrm{AU}$, an almost symmetric $20^{\circ}$ warp is apparent in Figure $1 d$. Total continuum flux densities are $22 \pm 4$ and $150 \pm 30 \mathrm{mJy}$ at 2.7 and $1.3 \mathrm{~mm}$, respectively. Thus, we recover completely the $1.3 \mathrm{~mm}$ flux of the unresolved component as measured with the IRAM $30 \mathrm{~m}$ telescope, $160 \mathrm{mJy}$ (Launhardt \& Henning 1997). From our maps, we calculate that about $50 \mathrm{mJy}$ arises in the envelope and $100 \mathrm{mJy}$ in the narrow central structure. Of this $100 \mathrm{mJy}$, about $75 \mathrm{mJy}$ derives from the inner 230 AU where the $3 \mathrm{~mm}$ emission arises.

In Figure 2, the $1.3 \mathrm{~mm}$ continuum MEM image is overlaid on a map of the NIR reflection nebula. Within the NIR astrometric uncertainties, 0 ." 5 , the dust emission coincides with the extinction lane and with the location and orientation of the disk postulated by Stecklum et al. (2001). Our kinematic data support the disk hypothesis. As illustrated in Figure $3 a$, strong ${ }^{13} \mathrm{CO}(1-0)$ emission $\left(T_{R}^{*} \sim 20 \mathrm{~K}\right)$ arises mainly from the outer parts of the disk, suggesting optically thick emission. In Figure $3 b$, the ${ }^{13} \mathrm{CO}$ line is clearly double-peaked, with blueshifted emission concentrated to the northeast and redshifted emission concentrated to the southwest. The velocity structure indicates a rotation axis perpendicular to the projected plane of the disk. At the systemic velocity, $v_{\mathrm{LSR}}=5.5 \mathrm{~km} \mathrm{~s}^{-1}$, gas emission appears to be self-absorbed, probably due to the extended outer envelope.

\section{DISCUSSION}

Our millimeter interferometric observations of thermal dust continuum and ${ }^{13} \mathrm{CO}$ line emission from $\mathrm{CB} 26$ confirm the 


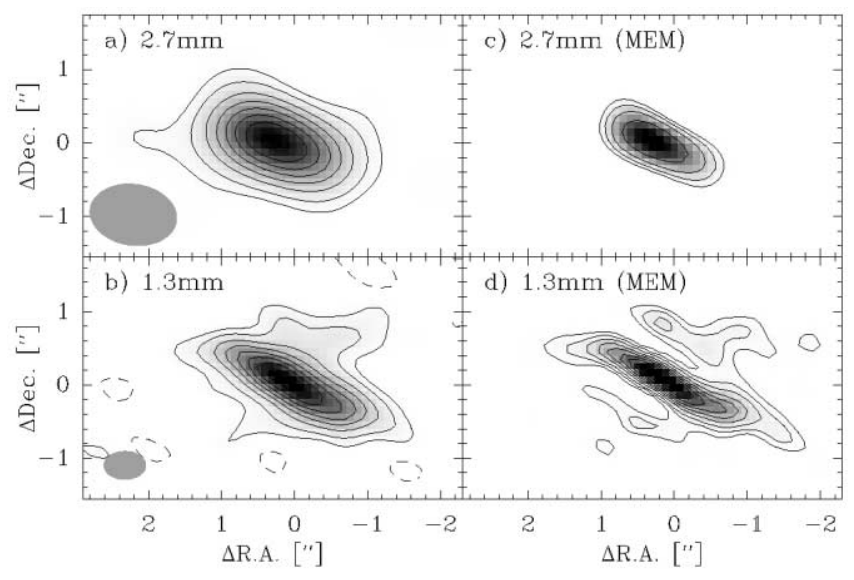

FIG. 1.-Dust continuum emission from CB 26 (R.A. $=04^{\mathrm{h}} 59^{\mathrm{m}} 50^{\mathrm{s}} 74$, decl. $=+52^{\circ} 04^{\prime} 43^{\prime \prime} .8$ [J2000]): $(a, b)$ Cleaned and restored 2.7 and $1.3 \mathrm{~mm}$ maps. Contour levels are at $-2,2,4,7, \ldots, 22 \times 1 \sigma \mathrm{rms}$, where $\sigma=0.7$ mJy beam ${ }^{-1}$ at $2.7 \mathrm{~mm}$ and $1.3 \mathrm{mJy}$ beam $^{-1}$ at $1.3 \mathrm{~mm}$. Beam sizes are shown as gray ovals $(1.2 \times 0.284$ at $2.7 \mathrm{~mm}$ and $0.58 \times 0.39$ at $1.3 \mathrm{~mm}) .(c, d)$ Deconvolved maps derived using the maximum entropy algorithm (MEM).

presence of an edge-on circumstellar disk as postulated by Stecklum et al. (2001). The disk has a diameter of about $400 \mathrm{AU}$ and an as yet unexplained warp beyond radii of 120 AU. This could be due to an undetected wide companion star with an inclined orbit.

The continuum fluxes lead to an average $1.3-3 \mathrm{~mm}$ dust emission spectral index $\alpha$ for the inner $R<115$ AU of the disk of $1.7 \pm 0.2$, where flux $S_{\nu} \propto \nu^{\alpha}$. Since $\alpha=2$ for a blackbody in the Rayleigh-Jeans limit, emission from the inner disk must be very optically thick, making it difficult to constrain the mass. Beyond $120 \mathrm{AU}$, no $3 \mathrm{~mm}$ emission is detectable, and $\alpha \geq$ $2.7 \pm 0.3$, suggesting optically thin emission. The transition between optically thick and optically thin emission occurs approximately at the radius where a warp appears, 120 AU. Thus, the outer disk may be disturbed and inflated, and flared $\mathrm{T}$ Tauri star (TTS) disk models may no longer apply. Nevertheless, we derived a lower limit to the disk mass by comparing the 1.3 and $2.7 \mathrm{~mm}$ continuum emission to spatially resolved radiative transfer calculations of different heuristic "typical" TTS disk models characterized by temperature profiles, $T_{d} \propto$ $r^{-q}$, with $q=0.5-0.75$, surface density profiles, $\Sigma \propto r^{-p}$, with $p=1.0-1.5$, and disk flaring, $H \propto r^{z}$, with $z=0-1.5$ (e.g., Adams et al. 1987; Kenyon \& Hartmann 1987). An interstellar gas-to-dust mass ratio of 100 and a typical disk dust opacity of $\kappa_{1.3}=2 \mathrm{~cm}^{2} \mathrm{~g}^{-1}$ with $\kappa \propto \nu^{\beta}$ and $\beta=1$ were assumed (see Beckwith et al. 1990). All models suggest disk masses of at least $0.1 M_{\odot}$. Due to the high optical depths of the ${ }^{13} \mathrm{CO}$ line, self-absorption, and possible depletion (see Thi et al. 2001), an independent mass estimate from the molecular line emission is unobtainable.

The envelope detected in the $1.3 \mathrm{~mm}$ images (Figs. $1 b$ and $1 d$ ) is probably associated with an outflow or disk wind. Assuming optically thin emission at temperature $30 \mathrm{~K}$ and $\kappa_{1.3}=1 \pm 0.5 \mathrm{~cm}^{2} \mathrm{~g}^{-1}$, a fairly typical value for dense protostellar cores (see Ossenkopf \& Henning 1994), we derive $M_{\mathrm{H}}(\mathrm{env})=0.01 \pm 0.003 M_{\odot}$. For a cylindrical morphology (see Figs. $1 b$ and $1 d$ and $\S 3$ ), we infer an average density $\left\langle n_{\mathrm{H}}\right\rangle \sim 3 \times 10^{8} \mathrm{~cm}^{-3}$, a column density $N_{\mathrm{H}} \sim 5 \times 10^{23} \mathrm{~cm}^{-2}$, and a corresponding visual extinction $A_{V} \sim 300 \mathrm{mag}$, implying that back-warming from the envelope has to be considered in the energy balance of the disk (see Butner, Natta, \& Evans 1994). The total mass of the more extended ( 3000 AU di-

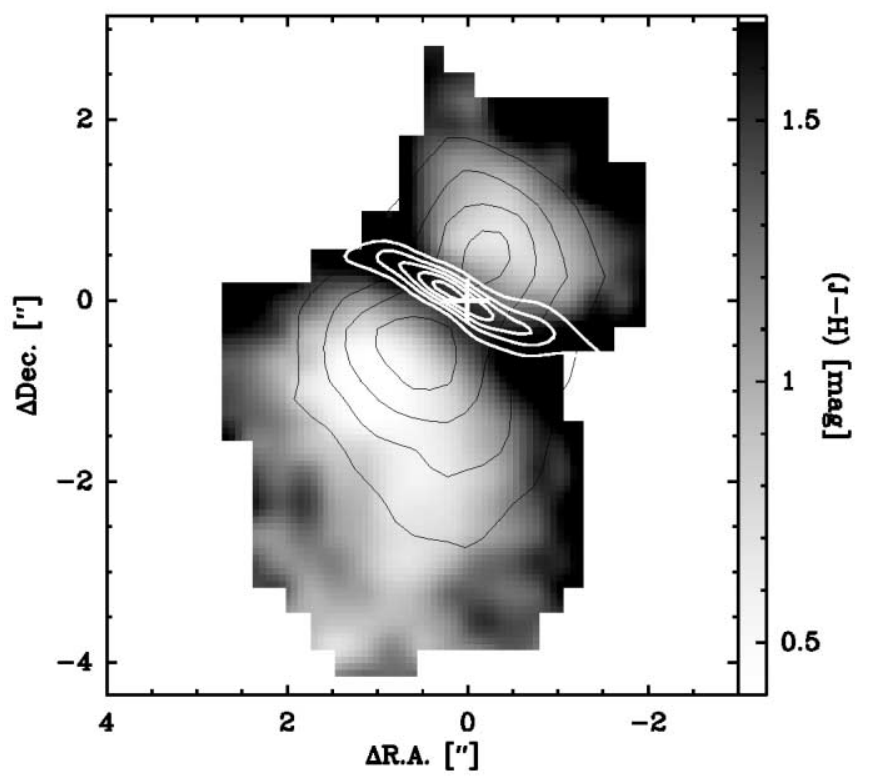

FIG. 2.-MEM image of the $1.3 \mathrm{~mm}$ dust emission (solid white contours at $4,11,18,25$, and $32 \mathrm{mJy} \operatorname{arcsec}^{-2}$ ) overlaid on a $J-H$ color map of the bipolar NIR reflection nebula. The black contours show the nebula $K$-band emission. The white cross denotes the presumed location of the illuminating star (from Stecklum et al. 2001).

ameter) asymmetric envelope seen in single-dish maps is $0.1 \pm 0.05 M_{\odot}$ (Henning et al. 2001). This may be a remnant of the globule core from which the system formed.

The velocity structure of ${ }^{13} \mathrm{CO}$ can be modeled by Keplerian rotation when the high optical depth of the inner disk and selfabsorption due to an extended envelope are taken into account, yielding a mass of the central star of $0.35 \pm 0.1 M_{\odot}$ (see

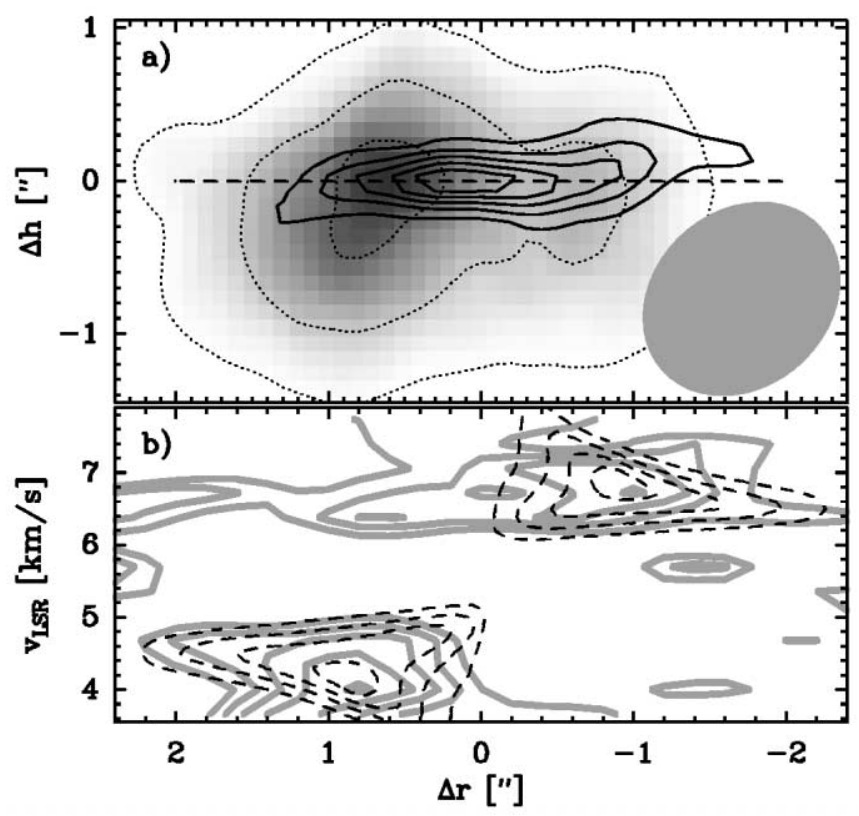

FIG. 3.-(a) Integrated ${ }^{13} \mathrm{CO}(1-0)$ emission from CB 26 in gray scale (dotted contours at $8,13.5$, and $19 \mathrm{~K} \mathrm{~km} \mathrm{~s}^{-1}$ ) with $1.3 \mathrm{~mm}$ dust continuum contours overlaid. A dashed line represents the plane of the disk. The CO beam size is shown as a gray ellipse. (b) Position-velocity diagram along the disk major axis. The thick contours at $27 \%, 45 \%, 63 \%, 81 \%$, and $99 \%$ of the maximum intensity show the observed velocity field. The expected emission from a Keplerian disk around a $0.35 M_{\odot}$ star is represented by dashed contours (see text). 
Fig. $3 b)$. However, since the disk mass $\left(\geq 0.1 M_{\odot}\right)$ is comparable to the inferred mass of the central star, self-gravitation may be important in the outer disk (see Bertin \& Lodato 1999). The outer rotation curve would then be flatter than in the pure Keplerian case, and the mass of the central star concomitantly lower, perhaps only $0.25 \mathrm{M}_{\odot}$. The ${ }^{13} \mathrm{CO}$ line strength suggests a gas-rich disk with kinetic temperature $\geq 20 \mathrm{~K}$ outside $R \sim$ $100 \mathrm{AU}$. An upper limit to the turbulent velocity dispersion in the disk is $0.3 \mathrm{~km} \mathrm{~s}^{-1}$.

PMS evolutionary tracks indicate an age of $(7 \pm 2) \times 10^{5} \mathrm{yr}$ for a $0.3 M_{\odot}$ star with $L=0.5 L_{\odot}$ (D'Antona \& Mazzitelli 1994). Due to the anisotropic radiation field caused by the optically thick edge-on disk, $0.5 L_{\odot}$ may be only a lower limit to the bolometric luminosity of the central star (see Men'shchikov, Henning, \& Fischer 1999). But, for such a massive disk, the intrinsic viscous luminosity could also contribute. Overall, the properties of the CB 26 system are consistent with its being younger than classical TTSs (ages $10^{6}-10^{7} \mathrm{yr}$ ). There is no dense, centrally peaked cloud core with evidence of collapse and no prominent molecular outflow, indicating that the main accretion phase, assumed to last a few times $10^{4} \mathrm{yr}$ (e.g., André, Ward-
Thompson, \& Barsony 2000), has ended. However, the disk mass is close to the theoretical gravitationally unstable limit, $0.3 M_{*}$, so that the envelope may still be accreting at low rates onto the disk (see Hollenbach, Yorke, \& Johnstone 2000 and references therein). Indeed, Figure $3 b$ shows some "forbidden" redshifted emission from the blue side of the disk that may be due to infall.

We conclude that the CB 26 disk surrounds a PMS M-type star with age $\sim 10^{5}$ yr. This represents an intermediate stage between such deeply embedded protostellar accretion disks such as L1551 IRS 5 (e.g., Butner et al. 1994) and the more evolved, perhaps protoplanetary, disks around classical TTSs, which may be the reservoirs for material for planet formation.

The Owens Valley millimeter-wave array is supported by NSF grant AST 99-81546. Funding from NASA's Origins of Solar Systems program (through grant NAG5-9530) is gratefully acknowledged. Research at Owens Valley on the formation of young stars and planets is also supported by the Norris Planetary Origins Project. We benefited from discussions with B. Stecklum, L. Hartmann, and H. Butner.

\section{REFERENCES}

Adams, F. C., Lada C. J., \& Shu, F. H. 1987, ApJ, 312, 788

André, P., Ward-Thompson, D., \& Barsony, M. 2000, in Protostars and Planets IV, ed. V. Mannings, A. P. Boss, \& S. S. Russell (Tucson: Univ. Arizona Press), 59

Beckwith, S. V. W., Sargent, A. I., Chini, R., \& Güsten, R. 1990, AJ, 99, 924 Bertin, G., \& Lodato, G. 1999, A\&A, 350, 694

Butner, H. M., Natta, A., \& Evans, N. J., II. 1994, ApJ, 420, 326

D’Antona, F., \& Mazzitelli, I. 1994, ApJS, 90, 467

Henning, Th., Wolf, S., Launhardt, R., \& Waters, R. 2001, ApJ, 561, 871

Hollenbach, D. J., Yorke, H. W., \& Johnstone, D. 2000, in Protostars and Planets IV, ed. V. Mannings, A. P. Boss, \& S. S. Russell (Tucson: Univ. Arizona Press), 401

Kenyon, S. J., \& Hartmann, L. 1987, ApJ, 323, 714
Kenyon, S. J., \& Hartmann, L. 1995, ApJS, 101, 117 Launhardt, R., \& Henning, Th. 1997, A\&A, 326, 329

Men'shchikov, A. B., Henning, Th., \& Fischer, O. 1999, ApJ, 519, 257

Ossenkopf, V., \& Henning, Th. 1994, A\&A, 291, 943

Sault, R. J., Teuben, P. J., \& Wright, M. C. H. 1995, in ASP Conf. Ser. 77, Astronomical Data Analysis Software and Systems IV, ed. R. A. Shaw, H. E. Payne, \& J. J. E. Hayes (San Francisco: ASP), 433

Scoville, N. Z., Carlstrom, J. E., Chandler, C. J. Phillips, J. A., Scott, S. L., Tilanus, R. P. J., \& Wang, Z. 1993, PASP, 105, 1482

Stecklum, B., Fischer, O., Launhardt, R., \& Leinert, Ch. 2001, ApJL, submitted Thi, W. F., et al. 2001, ApJ, 561, 1074

Ungerechts, H., \& Thaddeus, P. 1987, ApJS, 63, 645 